\title{
Abicipar pegol: the non-monoclonal antibody anti-VEGF
}

\author{
Ashish Sharma ${ }^{1} \cdot$ Nilesh Kumar $\mathbb{D}^{1} \cdot$ Baruch D. Kuppermann ${ }^{2} \cdot$ Francesco Bandello $^{3}$
}

Received: 10 September 2019 / Accepted: 13 September 2019 / Published online: 30 September 2019

(c) The Royal College of Ophthalmologists 2019

The introduction of anti-VEGF molecules has dramatically reduced blindness attributable to neovascular age-related macular degeneration (nAMD). These molecules however have a relatively short intraocular half-life, which requires frequent injections on a regular basis, typically ranging from 4 to 12 weeks when a treat and extend injection protocol is utilized [1]. The frequent dosing intervals can be a significant burden for the patient and their families, and can be a cause for discontinuation of the therapy.

The anti-VEGF clinical trials have demonstrated that the visual gains attained with current anti-VEGF monotherapy are +8 to +10 ETDRS letters. However, this significant benefit does not appear to improve with increased dosage or frequency of injections, thus indicating a possible therapeutic ceiling [2]. Trials such as PrONTO and SUSTAIN tried to extend the dosing interval while retaining the efficacy and were able to reduce the number of injections to 5.6 for 12 months of study; however, vision was negatively impacted [3, 4]. HORIZON trial suggests that less frequent injections may have an incremental decline in best-corrected visual acuity (BCVA) when compared to monthly injections of ranibizumab [5].

The next generation of anti-VEGF molecules are designed to provide a better dosing regimen with a sustained duration of action. Recently, brolucizumab, an $\mathrm{scFv}$ molecule has been filed for biologics license application (BLA) from FDA [6]. Faricimab is a unique bispecific mAb being developed on crossmAb platform to simultaneously inhibit Ang2 and VEGF and has been shown superior to

Ashish Sharma

drashish79@hotmail.com

1 Department of Vitreoretina, Lotus Eye Hospital and Institute, Coimbatore, TN, India

2 Gavin Herbert Eye Institute, University of California, Irvine, Irvine, CA, USA

3 Department of Ophthalmology, Scientific Institute San Raffaele, University Vita-Salute, Milano, Italy
anti-VEGF monotherapy with ranibizumab [7]. The most novel of all the molecules in pipeline for intravitreal therapy is Abicipar Pegol. (Allergan Inc, Switzerland) [1].

Abicipar Pegol is an anti-VEGF molecule based on the designed ankyrin repeat proteins (DARPin) therapeutics (Molecular Partners AG, Switzerland). DARPins are derived from naturally occurring ankyrin protein repeats (Fig. 1). The repeats are usually limited to four to six in numbers and thus lead to a right-handed solenoid structure with hydrophobic core and a large, grooved, solvent accessible binding surface [8]. Libraries of DARPin molecules of varying repeats numbers have been generated by a patented technology of protein engineering and recombinant DNA technology.

Early in-vivo and in-vitro trials identified few DARPin molecules that had the potential for ocular use. Intravitreal injections of DARPin in mouse models prevented retinal vascularization, while topical drops prevented corneal neovascularisation related to suture tracts in rabbits. It also reduced vascular leakage from retinal vessels in rabbits and prevented LASER induced choroidal neovascularisation in rats. It also had a favorable toxicity profile which led to the development of anti-VEGF DARPin molecule [1].

Abicipar was engineered as MP-0112 by Molecular Partners to have a longer ocular half-life with faster systemic clearance. Due to their low molecular weight and no immunoglobulin component, naive DARPins are rapidly cleared from the systemic circulation by kidneys. A polyethylene glycol tail was added to the repeat protein which helped the molecule to stay above renal clearance threshold $[8,9]$. It was the first DARPin molecule that entered clinical development with an aim to reduce the number of intravitreal injections needed.

Initial preclinical trials showed significantly longer half-life of the molecule (6 days) following intravitreal injections in rabbits compared with aflibercept (4.7 days) and ranibizumab (2.5 days). Phase 1 dose escalation study showed the maximum tolerated dose (MTD) to be $1 \mathrm{mg}$ with visual gains over the 16 weeks of study period in patients of nAMD. The trial also showed that at $2 \mathrm{mg}$ 


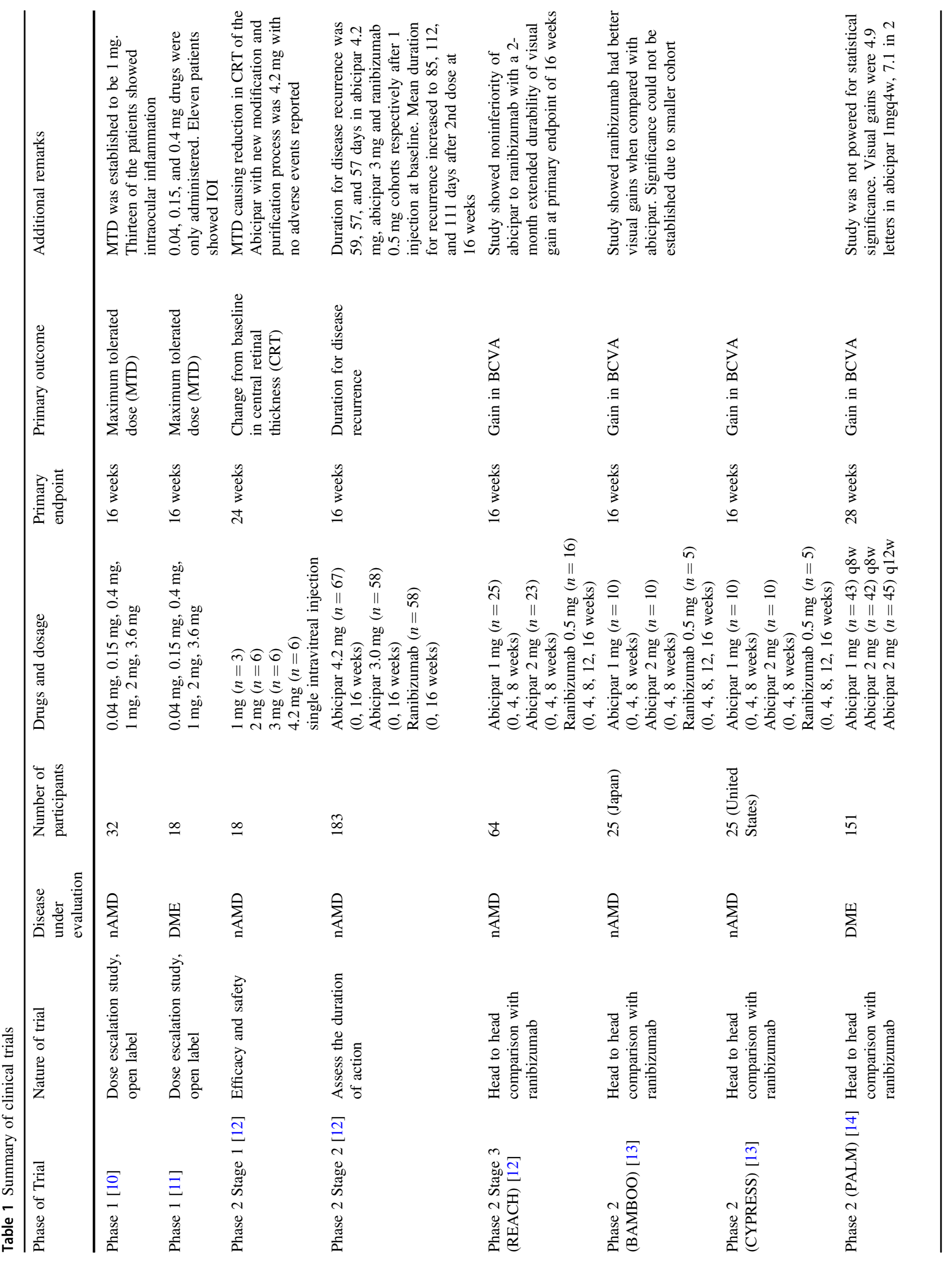




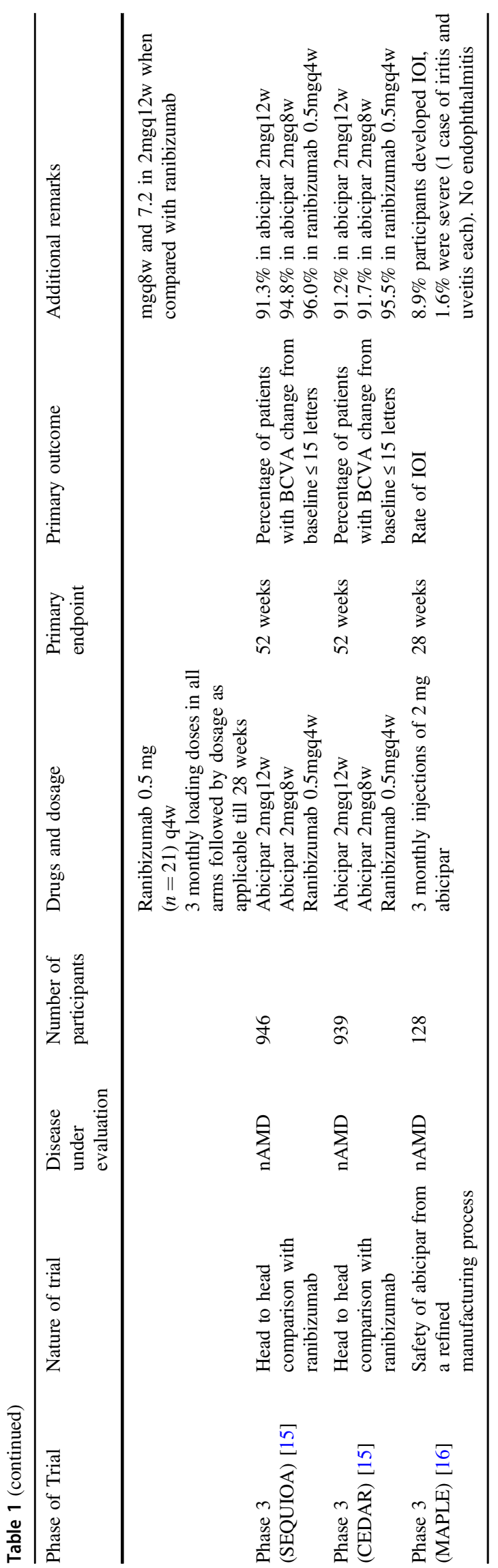

dosing, the systemic circulation of drug fell below detectable level in 2 weeks, indicating a reduced systemic exposure and antidrug antibody formation. Phase 1 trial with DME patients also showed improvement in BCVA and reduction in central subfield thickness up to $0.4 \mathrm{mg}$ dosing. Both trials showed high rate of inflammation attributable to the impurities in the drug formulation $[10,11]$.

The manufacturing process of the molecule was modified before the phase 2 trial was initiated, which was conducted in 3 phases for nAMD. The MTD for the new formulation was established at $4.2 \mathrm{mg}$ in the stage 1 trial. Stage 2 established the molecule to have a similar duration of action, when duration for disease recurrence was compared with ranibizumab. Stage $3 \mathrm{REACH}$ trial established the noninferiority of the molecule's 2-monthly dosing when compared with monthly regimen of ranibizumab [12].

Another smaller cohort phase 2 study compromised of two trials, BAMBOO and CYPRESS with 25 patients each which concluded ranibizumab to demonstrate higher visual gains when compared with abicipar [13]. The visual gains in ranibizumab arm were almost double than achieved in ANCHOR and MARINA trials, and thus the results were attributed to smaller cohort and concluded that the results cannot be applied over general population.

PALM trial, a phase 2 study on patients with DME was conducted on 151 patients. The study though was not powered to show statistical significance, it showed an improvement of 7.2 letters in $2 \mathrm{mgQ} 12 \mathrm{w}, 7.1$ letters in 2mgQ8w, 4.9 letters in $1 \mathrm{mgQ} 8 \mathrm{w}$, and 9.6 letters in ranibizumab $0.5 \mathrm{mg}$ monthly dosing regimen [14].

Phase 3 trials of abicipar for nAMD, dubbed as SEQUOIA and CEDAR, demonstrated similar efficacy of 2 mg abicipar in 6 and 8 injections compared to 13 injections of ranibizumab at 52 weeks primary end point in terms of visual gains. These phase 3 pivotal trials were the first to have a treatment group assigned at baseline to 12-week dosing (after a series of loading injections). The stability of visual gains in participants of q8w $(94.8 \%$ in SEQUOIA, 91.7\% in CEDAR) and $\mathrm{q} 12 \mathrm{w}(91.3 \%, 91.2 \%)$ dosing of abicipar was similar to that for participants of $\mathrm{q} 4 \mathrm{w}$ dosing of ranibizumab $(96.0 \%, 95.5 \%)$ [15].

The rates of intraocular inflammation (IOI) in both the trials were higher in patients receiving abicipar compared to subjects treated with ranibizumab. Investigation identified impurities, potentially from fragments of $E$. coli, an integral part of the manufacturing process of DARPins, in the formulation. This led the sponsors of the trials to modify the manufacturing process of abicipar which was evaluated in an open label 28 weeks trial for safety. MAPLE trial demonstrated a reduced IOI rate of $8.9 \%$ with $1.6 \%$ case of 
Fig. 1 Molecular structure of DARPin

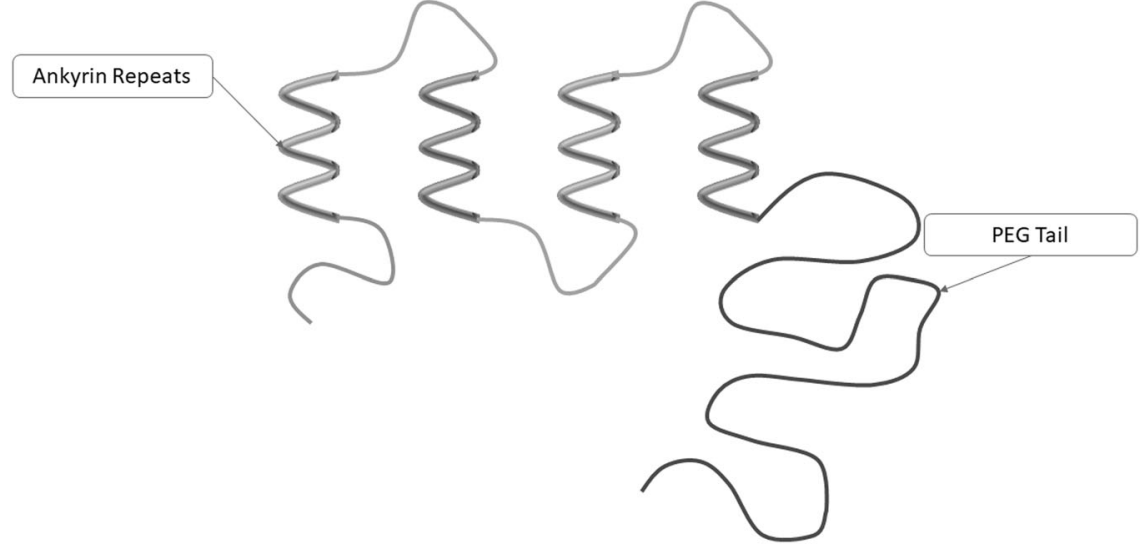

severe IOI, when compared with up to $16.3 \%$ in phase 2 . The phase 3 trials for DME are expected to be conducted with abicipar obtained from the modified manufacturing process [16].

Spurred by the positive outcomes of phase 3 trials in nAMD, the FDA has accepted a BLA and the European Medicines Agency has validated a marketing authorization application for abicipar pegol [17]. The promise of extended duration anti-VEGF treatment with q12 week apicipar dosing while maintaining good visual outcomes (and hopefully reduced rates of IOI with the new formulation) could usher in an a new era of decreased treatment burden to the benefit of patients and their families. Trials are summarized in Table 1.

\section{Compliance with ethical standards}

Conflict of interest Ashish Sharma: Consultant-Novartis India, Allergan Global, Intas India, Bayer India. Nilesh Kumar: None. BD Kuppermann: Clinical research-Alcon, Alimera, Allegro, Allergan, Apellis, Clearside, Genentech, GSK, Ionis, jCyte, Novartis, Regeneron, ThromboGenics; Consultant-Alimera, Allegro, Allergan, Cell Care, Dose, Eyedaptic, Galimedix, Genentech, Glaukos, Interface Biologics, jCyte, Novartis, Ophthotech, Regeneron, Revana, Theravance Biopharma. Francesco Bandello: Consultant-Allergan, Bayer, Boehringer- Ingelheim, Fidia Sooft, Hofmann La Roche, Novartis, NTC Pharma, Sifi, Thrombogenics, Zeiss.

Publisher's note Springer Nature remains neutral with regard to jurisdictional claims in published maps and institutional affiliations.

\section{References}

1. Stahl A, Stumpp MT, Schlegel A, Ekawardhani S, Lehrling C, Martin G, et al. Highly potent VEGF-A-antagonistic DARPins as anti-angiogenic agents for topical and intravitreal applications. Angiogenesis. 2013;16:101-11.

2. Smithwick E, Stewart MW. Designed ankyrin repeat proteins: a look at their evolving use in medicine with a focus on the treatment of chorioretinal vascular disorders. AIAAMC 2017; $16: 1-1$.
3. Lalwani GA, Rosenfeld PJ, Fung AE, Dubovy SR, Michels S, Feuer W, et al. A variable-dosing regimen with intravitreal ranibizumab for neovascular age-related macular degeneration: year 2 of the PrONTO study. Am J Ophthalmol. 2009;148:43-58.e1.

4. Holz FG, Amoaku W, Donate J, Guymer RH, Kellner U, Schlingemann RO, et al. Safety and efficacy of a flexible dosing regimen of ranibizumab in neovascular age-related macular degeneration: the SUSTAIN study. Ophthalmology. 2011;118:663-71.

5. Singer MA, Awh CC, Sadda S, Freeman WR, Antoszyk AN, Wong P, et al. HORIZON: an open-label extension trial of ranibizumab for choroidal neovascularization secondary to age-related macular degeneration. Ophthalmology. 2012;119:1175-83.

6. Novartis announces FDA filing acceptance and Priority Review of brolucizumab (RTH258) for patients with wet AMD. Novartis. 2019. https://www.novartis.com/news/media-releases/novartis-announcesfda-filing-acceptance-and-priority-review-brolucizumab-rth258patients-wet-amd

7. Klein C, Schaefer W, Regula JT, Dumontet C, Brinkmann U, Bacac M, et al. Engineering therapeutic bispecific antibodies using CrossMab technology. Methods. 2019;154:21-31.

8. Plückthun A. Designed ankyrin repeat proteins (DARPins): binding proteins for research, diagnostics, and therapy. Annu Rev Pharmacol Toxicol. 2015;55:489-511.

9. Sennhauser G, Grütter MG. Chaperone-assisted crystallography with DARPins. Structure. 2008;16:1443-53.

10. Souied EH, Devin F, Mauget-Faÿsse M, Koláŕ P, WolfSchnurrbusch U, Framme C, et al. Treatment of exudative agerelated macular degeneration with a designed ankyrin repeat protein that binds vascular endothelial growth factor: a phase I/II study. Am J Ophthalmol. 2014;158:724-732.e2.

11. Campochiaro PA, Channa R, Berger BB, Heier JS, Brown DM, Fiedler $\mathrm{U}$, et al. Treatment of diabetic macular edema with a designed ankyrin repeat protein that binds vascular endothelial growth factor: a phase I/II study. Am J Ophthalmol. 2013; 155:697-704.e2.

12. Callanan D, Kunimoto D, Maturi RK, Patel SS, Staurenghi G, Wolf S, et al. Double-masked, randomized, phase 2 evaluation of abicipar pegol (an Anti-VEGF DARPin Therapeutic) in neovascular age-related macular degeneration. J Ocul Pharmacol Ther. 2018;34:700-9.

13. Kunimoto D, Ohji M, Maturi RK, Sekiryu T, Wang Y, Pan G, et al. Evaluation of Abicipar Pegol (an Anti-VEGF DARPin Therapeutic) in patients with neovascular age-related macular degeneration: studies in Japan and the United States. Ophthalmic Surg Lasers Imaging Retina 2019;50:e10-22.

14. Abicipar Pegol PALM Study Phase 2 Data in Diabetic Macular Edema (DME) Presented at 2016 AAO Annual Meeting- 
Molecular Partners. 2016. https://www.molecularpartners.com/a bicipar-pegol-palm-study-phase-2-data-in-diabetic-macularedema-dme-presented-at-2016-aao-annual-meeting/

15. Allergan and Molecular Partners Announce Two Positive Phase 3 Clinical Trials for Abicipar pegol 8 and 12-week Regimens for the Treatment in Patients with Neovascular Age-Related Macular Degeneration-Molecular Partners. 2018. https://www.molecula rpartners.com/allergan-and-molecular-partners-announce-twopositive-phase-3-clinical-trials-for-abicipar-pegol-8-and-12-weekregimens-for-the-treatment-in-patients-with-neovascular-age-rela ted-macular-degeneration/
16. Allergan and Molecular Partners Announce Topline Safety Results from MAPLE study of Abicipar pegol—Molecular Partners. 2019. https://www.molecularpartners.com/allergan-and-molecular-pa rtners-announce-topline-safety-results-from-maple-study-of-abiciparpegol/

17. FDA, European Medicines Agency advance abicipar pegol applications for wet AMD. 2019. https://www.healio.com/ophtha lmology/retina-vitreous/news/online/\%7B401f1470-71d1-4ebd907e-5f13e0b9cd99\%7D/fda-european-medicines-agency-adva nce-abicipar-pegol-applications-for-wet-amd 